\title{
GENERALIZED CLUSTER TILTING OF $n$-ABELIAN CATEGORIES
}

\section{DEREN LUO, ZHE LI and DALONG YANG}

College of Mathematics

Hunan Institute of Science and Technology

414006 Yueyang

P. R. China

e-mail: luoderen@126.com

\begin{abstract}
In this paper, we study the $(m, n)$-cluster tilting subcategories of $n$-abelian categories as a generalization of $m$-cluster tilting subcategories of abelian categories and prove that the $(m, n)$-cluster tilting subcategories of certain $n$-abelian categories are $m n$-abelian categories.
\end{abstract}

2020 Mathematics Subject Classification: 18G50, 18G15, $18 \mathrm{E} 25$.

Keywords and phrases: $n$-abelian category, $n$-derived functor, cluster tilting.

Received February 11, 2020; Revised March 7, 2020

(C) 2020 Scientific Advances Publishers

This work is licensed under the Creative Commons Attribution International License (CC BY 3.0).

http://creativecommons.org/licenses/by/3.0/deed.en_US

Open Access (cc) (1)




\section{Introduction}

In recent years, $n$-cluster tilting theory of representation theory of finite dimensional algebras, abelian categories, triangulated categories, derived categories comes into focus [1, 2, 3, 4, 7]. Motivated by these, Jasso introduced the $n$-abelian categories and $n$-exact categories [5] as a generalization of the classical abelian categories and exact categories, he proved that $n$-cluster tilting subcategories of abelian categories are $n$-abelian categories. As a generalization of homological theory of abelian categories, homological properties of $n$-abelian categories were introduced in $[8,9]$ via higher (co)homology of $n$-(co)resolutions under right (left) exact functors for $n$-exact sequences.

In this paper, we study the $(m, n)$-cluster tilting subcategories of $n$-abelian categories via $n$-homological theory of abelian categories as a generalization of $m$-cluster tilting subcategories of abelian categories.

This paper is organized as follows. In Section 2, we recall some notions and notations of (co)homology properties of $n$-abelian categories. In Section 3, we introduce the $(m, n)$-cluster tilting subcategories of certain $n$-abelian categories, and show that the $(m, n)$-cluster-tilting subcategories of $n$-abelian categories are $m n$-abelian categories, and study the relationship between $n m$-exact sequences and $m$-fold $n$-exact sequences.

\section{Definitions and Preliminaries}

\section{1. $n$-Abelian categories}

Let $n$ be a positive integer and $\mathcal{C}$ be an additive category. We denote the category of cochain complexes of $\mathcal{C}$ by $\mathrm{Ch}(\mathcal{C})$ and the homotopy category of $\mathcal{C}$ by $\mathrm{H}(\mathcal{C})$. Also, we denote by $\mathrm{Ch}^{n}(\mathcal{C})$ the full subcategory of $\mathrm{Ch}(\mathcal{C})$ given by all complexes

$$
X^{0} \stackrel{d^{0}}{\rightarrow} X^{1} \stackrel{d^{1}}{\rightarrow} \cdots \stackrel{d^{n-1}}{\rightarrow} X^{n} \stackrel{d^{n}}{\rightarrow} X^{n+1}
$$

which are concentrated in degrees $0,1, \ldots, n+1$. We write $\mathcal{C}(X, Y)$ for the morphisms in $\mathcal{C}$ from $X$ to $Y$, if $X, Y \in$ obC. 
Let $d^{0}: X^{0} \rightarrow X^{1}$ be a morphism in $\mathcal{C}$. An $n$-cokernel of $d^{0}$ is a sequence of morphisms

$$
\left(d^{1}, \ldots, d^{n}\right): X^{1} \stackrel{d^{1}}{\rightarrow} X^{2} \stackrel{d^{2}}{\rightarrow} X^{3} \rightarrow \cdots \stackrel{d^{n}}{\rightarrow} X^{n+1}
$$

such that for all $1 \leq k \leq n-1$ the morphism $d^{k}$ is a weak cokernel of $d^{k-1}$, and $d^{n}$ is moreover a cokernel of $d^{n-1}$. In this case, we say the sequence

$$
\left(d^{0}, d^{1}, \ldots, d^{n}\right): X^{0} \stackrel{d^{0}}{\rightarrow} X^{1} \stackrel{d^{1}}{\rightarrow} \cdots \stackrel{d^{n}}{\rightarrow} X^{n+1}
$$

is right n-exact. The concepts of $n$-kernel of a morphism and left n-exact are defined dually. If $n \geq 2$, the $n$-cokernels and $n$-kernels are not unique in general, but their are unique up to isomorphism in $H(\mathcal{C})$ [5]. (1) is called an n-exact sequence if it is both right $n$-exact and left $n$-exact. A sequence

$$
X^{0} \rightarrow X^{1} \rightarrow \cdots \rightarrow X^{m n+1}
$$

is called an $m$-fold $n$-exact sequence if it can be split into $m n$-exact sequences $Y^{i n} \rightarrow X^{i n+1} \rightarrow \cdots \rightarrow X^{(i+1) n} \rightarrow Y^{(i+1) n}$ for $i=0,1, \cdots, m-1$ where $Y^{0}=X^{0}$ and $Y^{m n}=X^{m n+1}$.

As a generalization of the notion of classical abelian categories, Jasso introduced the $n$-abelian categories in [5] as follows.

Definition 2.1 ( $n$-abelian category, Definition 3.1, [5]). An $n$-abelian category is an additive category $\mathcal{A}$ which satisfies the following axioms:

(A0) The category $\mathcal{A}$ is idempotent complete.

(A1) Every morphism in $\mathcal{A}$ has an $n$-kernel and an $n$-cokernel. 
(A2) For every monomorphism $f^{0}: X^{0} \rightarrow X^{1}$ in $\mathcal{A}$ there exists an $n$-exact sequence:

$$
X^{0} \stackrel{f^{0}}{\rightarrow} X^{1} \stackrel{f^{1}}{\rightarrow} \cdots \stackrel{f^{n-1}}{\rightarrow} X^{n} \stackrel{f^{n}}{\rightarrow} X^{n+1}
$$

$\left(\mathrm{A} 2^{o p}\right)$ For every epimorphism $f^{n}: X^{n} \rightarrow X^{n+1}$ in $\mathcal{A}$ there exists an $n$-exact sequence:

$$
X^{0} \stackrel{f^{0}}{\rightarrow} X^{1} \stackrel{f^{1}}{\rightarrow} \cdots \stackrel{f^{n-1}}{\rightarrow} X^{n} \stackrel{f^{n}}{\rightarrow} X^{n+1} .
$$

Note that 1-abelian categories are precisely abelian categories in the usual sense.

Let $\mathcal{A}$ be an abelian category and $\mathcal{D}$ be a generating-cogenerating full subcategory of $\mathcal{A}$. $\mathcal{D}$ is called an $n$-cluster-tilting subcategory of $\mathcal{A}$ if $\mathcal{D}$ is functorially finite in $\mathcal{A}$ and

$$
\begin{aligned}
\mathcal{D} & =\left\{X \in \mathcal{A} \mid \forall i \in\{1, \ldots, n-1\} \operatorname{Ext}_{\mathcal{A}}^{i}(X, \mathcal{D})=0\right\} \\
& =\left\{X \in \mathcal{A} \mid \forall i \in\{1, \ldots, n-1\} \operatorname{Ext}_{\mathcal{A}}^{i}(\mathcal{D}, X)=0\right\} .
\end{aligned}
$$

Note that $\mathcal{A}$ itself is the unique 1-cluster-tilting subcategory of $\mathcal{A}$.

Lemma 2.2 (Theorem 3.16, [5]). Let $\mathcal{A}$ be an abelian category and $\mathcal{D}$ be an n-cluster tilting subcategory of $\mathcal{A}$. Then, $\mathcal{D}$ is an n-abelian category.

\section{2. (co)Homology of $\boldsymbol{n}$-abelian categories}

In this subsection, we recall the right (resp., left) derived functors of covariant or contravariant left (resp., right) $n$-exact functors and study their basic properties. 
Let $\mathcal{A}$ be an $n$-abelian category and $\mathcal{B}$ be an abelian category, and let $G: \mathcal{A} \rightarrow \mathcal{B}$ be a covariant additive functor. Let $X: X^{0} \stackrel{d^{0}}{\rightarrow} X^{1} \stackrel{d^{1}}{\rightarrow} \cdots \stackrel{d^{n-1}}{\rightarrow}$ $X^{n} \stackrel{d^{n}}{\rightarrow} X^{n+1}$ in $\operatorname{Ch}^{n}(\mathcal{A})$ be an $n$-exact sequence. We say that $G$ is

(i) left $n$-exact if $0 \rightarrow G X^{0} \rightarrow G X^{1} \rightarrow \cdots \rightarrow G X^{n} \rightarrow G X^{n+1}$ is an exact sequence of $\mathcal{B}$.

(ii) right $n$-exact if $G X^{0} \rightarrow G X^{1} \rightarrow \cdots \rightarrow G X^{n} \rightarrow G X^{n+1} \rightarrow 0$ is an exact sequence of $\mathcal{B}$.

(iii) n-exact if $0 \rightarrow G X^{0} \rightarrow G X^{1} \rightarrow \cdots \rightarrow G X^{n} \rightarrow G X^{n+1} \rightarrow 0$ is an exact sequence of $\mathcal{B}$.

The notions of covariant (contravariant) additive left (right) $n$-exact functors are defined dually. For example, the hom-functors $\mathcal{A}(M,-)$ (resp., $\mathcal{A}(-, M)$ ) is covariant (resp., contravariant) left $n$-exact by the definition of $n$-kernel (resp., $n$-cokernel).

We say that an $n$-abelian category $\mathcal{A}$ has enough projectives if for every object $M \in \mathcal{A}$, there exist projective objects $P_{1}, P_{2}, \ldots, P_{n} \in \mathcal{A}$ and an $n$-exact sequence $N \rightarrow P_{n} \rightarrow \cdots \rightarrow P_{1} \rightarrow M$. The notion of having enough injectives is defined dually. Let $\mathcal{A}$ has enough projectives, $M \in \mathcal{A}$, there are $n$-exact sequences

$$
\begin{aligned}
& \Omega_{n} M \stackrel{j_{1}}{\rightarrow} P_{n} \stackrel{d_{n}}{\rightarrow} \cdots \rightarrow P_{1} \rightarrow M \\
& \Omega_{n}^{2} M \stackrel{j_{2}}{\rightarrow} P_{2 n} \stackrel{d_{2 n}}{\rightarrow} \cdots \rightarrow P_{n+1} \stackrel{\pi_{1}}{\rightarrow} \Omega_{n} M .
\end{aligned}
$$

Connecting them, let $d_{i n+1}=j_{i} \pi_{i}$, we call the sequence

$$
\cdots \rightarrow P_{3 n} \stackrel{d_{3 n}}{\rightarrow} \cdots \rightarrow P_{2 n+1} \stackrel{d_{2 n+1}}{\rightarrow} P_{2 n} \stackrel{d_{2 n}}{\rightarrow} \cdots \rightarrow P_{n+1} \stackrel{d_{n+1}}{\rightarrow} P_{n} \stackrel{d_{n}}{\rightarrow} \cdots \rightarrow P_{1} \stackrel{d_{1}}{\rightarrow} M
$$


a projective $n$-resolution of $M$, also denoted simply as $P \bullet \stackrel{d_{1}}{\rightarrow} M$. We call $\Omega_{n}^{k} M$ the $k$-th $n$-syzygy of $M$ for $k \geq 0$. The notions of injective $n$-resolution, $k$-th n-cosyzygy $\Omega_{n}^{-k} M$ of $M$ are defined dually.

Let $F: \mathcal{A} \rightarrow \mathcal{B}$ be a contravariant left $n$-exact functor. The right $n$-derived functors $\mathrm{nR}^{i} F$ for $i \geq 0$ as follows, for any $M \in \mathcal{A}$, choose a projective $n$-resolution $P_{\bullet} \rightarrow M$ as (2) and define

$$
\mathrm{nR}^{i} F(M):=H_{i n+1}(F P \bullet):=\operatorname{Ker} F d_{i n+2} / \operatorname{Im} G d_{i n+1} \text { for } i=0,1, \cdots .
$$

Note that $\mathrm{nR}^{0} F(M) \simeq F M . \mathrm{nR}^{i} F(-)$ is an additive functor from $\mathcal{A}$ to $\mathcal{B}$ and $\mathrm{nR}^{i} F(P)=0$ for all projective object $P$ for any $i>0$. The notions of right (left) $n$-derived functors of covariant or contravariant left (right) $n$-exact functors are defined dually. Specially, for contravariant (resp., covariant) left $n$-exact functor $\mathcal{A}(-, B)($ resp., $\mathcal{A}(A,-))$, we define the right $n$-derived functors

$$
\mathrm{nExt}_{\mathcal{A}}^{i}(-, B)=\mathrm{nR}^{i} \mathcal{A}(-, B) \quad \text { resp., } \mathrm{nExt}_{\mathcal{A}}^{i}(A,-)=\mathrm{nR}^{i} \mathcal{A}(A,-) .
$$

In particular, $\mathrm{nExt}_{\mathcal{A}}^{0}(-, B)=\mathcal{A}(-, B), \mathrm{nExt}_{\mathcal{A}}^{0}(A,-)=\mathcal{A}(A,-)$.

There is an isomorphism $\mathrm{nE}^{m}(A, B) \cong \mathrm{nExt}_{\mathcal{A}}^{m}(A, B)$, here $\mathrm{nE}^{m}(A, B)$ is the equivalence classes of $m$-fold $n$-extensions of $A$ by $B$, it is an abelian group under $n$-Baer sum [8]. So, we can define $\mathrm{nExt}_{\mathcal{A}}^{m}(A, B)$ even without of projective objects and injective objects.

Lemma 2.3 ([8], Proposition 4.3). Let $\mathcal{A}$ be an n-abelian category, $A, B \in \mathcal{A}$, we have

(i) $n \operatorname{Ext}_{\mathcal{A}}^{i}(A,-)(B) \simeq n \operatorname{Ext}_{\mathcal{A}}^{i}(-, B)(A)=n \operatorname{Ext}_{\mathcal{A}}^{i}(A, B)$. 
(ii) If $\mathcal{A}$ is an $n$-cluster tilting subcategory of a projectively generated injectivity cogenerated abelian category $\mathcal{D}$. Then $n \operatorname{Ext}_{\mathcal{A}}^{m}(A, B) \simeq \operatorname{Ext}_{\mathcal{D}}^{m n}(A, B)$, $\operatorname{Ext}_{\mathcal{D}}^{m n+i}(A, B)=0 \forall A, B \in \mathcal{A}, m \geq 0,1 \leq i \leq n-1$.

(iii) $A$ is a projective object if and only if $\mathcal{A}(A,-)$ is an exact functor if and only if $n \operatorname{Ext}_{\mathcal{A}}^{i}(A, B)=0$ for all $i \neq 0$ and all $B$ if and only if $n \operatorname{Ext}_{\mathcal{A}}^{1}(A, B)=0$ for all $B$.

If an $n$-abelian category $\mathcal{A}$ is injectively cogenerated, then by the results of Jasso and Kvamme [5, 6], it follows that $\mathcal{A}$ is equivalent to an $n$-cluster tilting subcategory in the dual of the category of finitely presented covariant functors over the full subcategory of injective objects of $\mathcal{A}$ which is an injectively cogenerated abelian category.

Lemma 2.4. An injectively cogenerated additive category $\mathcal{C}$ is an $n$-abelian category if and only if there exists an injectively-cogenerated abelian categories $\mathcal{A}$ such that $\mathcal{C}$ can be embedded to $\mathcal{A}$ as an n-cluster tilting subcategory.

Using the Lemma 2.4, we can generalize the "Long $n$-exact sequence Theorem 4.5" of [8] as following:

Lemma 2.5. Let $\mathcal{A}$ be an injectively cogenerated n-abelian category. $X: X^{0} \stackrel{\alpha^{0}}{\rightarrow} X^{1} \stackrel{\alpha^{1}}{\rightarrow} \cdots \stackrel{\alpha^{n}}{\rightarrow} X^{n+1}$ an $n$-exact sequence of $\mathcal{A}$, for any object $A \in \mathcal{A}$, we have exact sequences

$$
\begin{aligned}
& 0 \rightarrow \mathcal{A}\left(A, X^{0}\right) \rightarrow \cdots \rightarrow \mathcal{A}\left(A, X^{n+1}\right) \stackrel{\partial_{n}}{\rightarrow} n \operatorname{Ext}_{\mathcal{A}}^{1}\left(A, X^{0}\right) \rightarrow \cdots \rightarrow n \operatorname{Ext}_{\mathcal{A}}^{1} \\
&\left(A, X^{n+1}\right) \stackrel{\partial_{n}^{1}}{\rightarrow} \cdots \stackrel{\partial_{n}^{i-1}}{\rightarrow} n \operatorname{Ext}_{\mathcal{A}}^{i}\left(A, X^{0}\right) \rightarrow \cdots \rightarrow n \operatorname{Ext}_{\mathcal{A}}^{i}\left(A, X^{n+1}\right) \stackrel{\partial_{n}^{i}}{\rightarrow} \cdots \\
& 0 \rightarrow \mathcal{A}\left(X^{n+1}, A\right) \rightarrow \cdots \rightarrow \mathcal{A}\left(X^{0}, A\right) \stackrel{\partial_{n}}{\rightarrow} n \operatorname{Ext}_{\mathcal{A}}^{1}\left(X^{n+1}, A\right) \rightarrow \cdots \rightarrow n E x t_{\mathcal{A}}^{1} \\
&\left(X^{0}, A\right) \stackrel{\partial_{n}^{1}}{\rightarrow} \cdots \stackrel{\partial_{n}^{i-1}}{\rightarrow} n \operatorname{Ext}_{\mathcal{A}}^{i}\left(X^{n+1}, A\right) \rightarrow \cdots \rightarrow n \operatorname{Ext}_{\mathcal{A}}^{i}\left(X^{0}, A\right) \stackrel{\partial_{n}^{i}}{\rightarrow} \cdots
\end{aligned}
$$




\section{Cluster Tilting Subcategories of $n$-Abelian Categories}

By Lemma 2.2, any $n$-cluster tilting subcategory of abelian category is an $n$-abelian category. It is natural to define $(m, n)$-cluster tilting subcategories of $n$-abelian categories.

Definition 3.1. Let $\mathcal{A}$ be an $n$-abelian category and $\mathcal{D}$ be a generating-cogenerating full subcategory of $\mathcal{A}$. $\mathcal{D}$ is called an $(m, n)$-cluster tilting subcategory of $\mathcal{A}$ if $\mathcal{D}$ is functorially finite in $\mathcal{A}$ and

$$
\begin{aligned}
\mathcal{D} & =\left\{X \in \mathcal{A} \mid \forall i \in\{1, \ldots, m-1\} \mathrm{nExt}_{\mathcal{A}}^{i}(X, \mathcal{D})=0\right\} \\
& =\left\{X \in \mathcal{A} \mid \forall i \in\{1, \ldots, m-1\} \mathrm{nExt}_{\mathcal{A}}^{i}(\mathcal{D}, X)=0\right\} .
\end{aligned}
$$

Note that $\mathcal{A}$ itself is the unique $(1, n)$-cluster-tilting subcategory of $\mathcal{A}$.

Let $\mathcal{A}$ be an abelian category, $\mathcal{D}$ be an $n$-cluster tilting subcategory of $\mathcal{A}$, and $\mathcal{M}$ be an $(m, n)$-cluster tilting subcategory of $\mathcal{D}$. Then, if $m=n=1, \quad \mathcal{A}=\mathcal{D}=\mathcal{M}$. If $n=1, m>1, \mathcal{M}$ is an $m$-cluster tilting subcategory of $\mathcal{A}$. If $n>1, m=1, \mathcal{M}$ is an $n$-cluster tilting subcategory of $\mathcal{A}$.

Our next aim is to show that the $(m, n)$-cluster tilting subcategories of small $n$-abelian categories are $m n$-abelian categories under certain conditions as a generalization of Lemma 2.2. First, we generalize ([5], Propositions 3.17, 3.18).

Lemma 3.2. Let $\mathcal{A}$ be an injectively cogenerated n-abelian category, $\mathcal{M}$ be an $(m, n)$-cluster tilting subcategory of $\mathcal{A}$. Then, for all $A \in \mathcal{A}$, there exist $n$-exact sequences,

$$
\begin{aligned}
& A \stackrel{f^{0}}{\rightarrow} M^{1} \rightarrow \cdots \rightarrow M^{n} \stackrel{g^{1}}{\rightarrow} D^{1} \\
& D^{1} \stackrel{f^{1}}{\rightarrow} M^{n+1} \rightarrow \cdots \rightarrow M^{2 n} \stackrel{g^{2}}{\rightarrow} D^{2} \\
& \quad \cdots \\
& D^{m-2} \stackrel{f^{m-2}}{\rightarrow} M^{(m-2) n+1} \rightarrow \cdots \rightarrow M^{(m-1) n} \rightarrow M^{(m-1) n+1}
\end{aligned}
$$


satisfying the following properties:

(i) $M^{i} \in \mathcal{M}$;

(ii) $f^{i}$ are left $\mathcal{M}$-approximations;

(iii) For all $M \in \mathcal{M}$, the induced sequence of abelian groups

$0 \rightarrow \mathcal{A}\left(M^{(m-1) n+1}, M\right) \rightarrow \mathcal{A}\left(M^{(m-1) n}, M\right) \rightarrow \cdots \rightarrow \mathcal{A}\left(M^{1}, M\right) \rightarrow \mathcal{A}(A, M) \rightarrow 0$

is exact.

Proof. This proof is an adaptation of the proof of ([5], Proposition 3.17). Note that $D^{0}=A$.

The existences of these $n$-exact sequences follow from the functorially finiteness of $\mathcal{M}$. Indeed, for any $A \in \mathcal{A}$, there exists a left $\mathcal{M}$-approximation $f^{0}: A \rightarrow M^{1}$. Since $\mathcal{A}$ is $n$-abelian, there exists a weak cokernel $k^{1}: M^{1} \rightarrow C^{1}$ in $\mathcal{A}$, then taking a left $\mathcal{M}$-approximation $t^{1}: C^{1} \rightarrow M^{2}$, this constructs a weak cokernel $t^{1} k^{1}$ of $f^{0}$. Inductively, we can construct a $n$-exact sequence $A \stackrel{f^{0}}{\rightarrow} M^{1} \rightarrow \cdots \rightarrow M^{n} \stackrel{g^{1}}{\rightarrow} D^{1}$, where $g^{1}$ is a cokernel of $M^{n-1} \rightarrow M^{n}$ by ([5], Proposition 3.7). Inductively, we can construct the desired $n$-exact sequences.

Given that for all $k \in\{0, \ldots, m-2\}$ the morphism $f^{k}$ is a left $\mathcal{M}$-approximation, it readily follows that the sequence

$$
0 \rightarrow \mathcal{A}\left(M^{(m-1) n+1}, M\right) \rightarrow \cdots \rightarrow \mathcal{A}\left(M^{1}, M\right) \rightarrow \mathcal{A}(A, M) \rightarrow 0
$$

is exact. It remains to show that $M^{(m-1) n+1} \in \mathcal{M}$. 
We claim that for each $M \in \mathcal{M}, \mathrm{nExt}_{\mathcal{A}}^{i}\left(M^{(m-1) n+1}, M\right)=0$ for $i \in\{1,2, \cdots, m-1\}$. First, note that for all $M \in \mathcal{M}$ applying the contravariant functor $\mathcal{A}(-, M)$ to the $n$-exact sequence (3), we have isomorphisms

$$
\operatorname{nExt}_{\mathcal{A}}^{i}\left(M^{(m-1) n+1}, M\right) \simeq \operatorname{nExt}_{\mathcal{A}}^{i-1}\left(D^{m-2}, M\right) \simeq \cdots \simeq \operatorname{nExt}_{\mathcal{A}}^{1}\left(D^{m-i}, M\right),
$$

for $i \in\{1,2, \cdots, m-1\}$ by long $n$-exact sequence theorem. Moreover, the morphism $\mathcal{A}\left(M^{(m-i-1) n+1}, M\right) \rightarrow \mathcal{A}\left(D^{m-i-1}, M\right)$ is an epimorphism for $f^{m-i-1}$ is a left $\mathcal{M}$-approximation of $\mathcal{A}$. Thus we have $\mathrm{nExt}_{\mathcal{A}}^{i}\left(M^{(m-1) n+1}, M\right)=0$ as required.

Lemma 3.3. Let $\mathcal{A}$ be an injectively cogenerated n-abelian category. Let $B \in \mathcal{A}$, and $\mathcal{M}$ be a subcategory of $\mathcal{A}$ such that $n \operatorname{Ext}_{\mathcal{A}}^{k}(\mathcal{M}, B)=0$ for all $k \in\{1, \ldots, m-1\}$. Consider a composition of $n$-exact sequences

$$
\begin{aligned}
& A_{1} \stackrel{f_{1}}{\rightarrow} M_{n} \rightarrow \cdots \rightarrow M_{1} \stackrel{g_{0}}{\rightarrow} A \\
& A_{2} \stackrel{f_{2}}{\rightarrow} M_{2 n} \rightarrow \cdots \rightarrow M_{n+1} \stackrel{g_{1}}{\rightarrow} A_{1} \\
& \vdots \\
& A_{m} \stackrel{f_{m}}{\rightarrow} M_{m n} \rightarrow \cdots \rightarrow M_{(m-1) n+1} \stackrel{g_{m-1}}{\rightarrow} A_{m-1}
\end{aligned}
$$

in $\mathcal{A}$ such that $M_{k} \in \mathcal{M}$ for all $k \in\{1,2, \ldots, m n\}$. Then, for each $k \in\{1, \ldots, m-1\}$ there is an isomorphism between $n \operatorname{Ext}_{\mathcal{A}}^{k}(A, B)$ and the cohomology of the induced complex

$$
\mathcal{A}\left(M_{1}, B\right) \rightarrow \mathcal{A}\left(M_{2}, B\right) \rightarrow \cdots \rightarrow \mathcal{A}\left(M_{m n}, B\right) \rightarrow \mathcal{A}\left(A_{m}, B\right)
$$

at $\mathcal{A}\left(M_{k n+1}, B\right)$. 
Proof. Note that $A_{0}=A$. First, let us show that for each $k \in\{1, \ldots, m-1\}$ there exist isomorphisms

$$
\mathrm{nExt}_{\mathcal{A}}^{k}\left(A_{0}, B\right) \simeq \mathrm{nExt}_{\mathcal{A}}^{k-1}\left(A_{1}, B\right) \simeq \cdots \simeq \mathrm{nExt}_{\mathcal{A}}^{1}\left(A_{k-1}, B\right) .
$$

The case $k=1$ is obvious. If $2 \leq k \leq m-1$, then for each $2 \leq \ell \leq k$ applying the functor $\mathcal{A}(-, B)$ to the exact sequence $0 \rightarrow A_{k-\ell+1} \rightarrow$ $M_{(k-\ell+1) n} \rightarrow \cdots \rightarrow M_{(k-\ell) n+1} \rightarrow A_{k-\ell} \rightarrow 0$ yields an exact sequence

$$
\begin{aligned}
0=\mathrm{nExt}_{\mathcal{A}}^{\ell-1}\left(M_{(k-\ell+1) n}, B\right) \rightarrow \mathrm{nExt}_{\mathcal{A}}^{\ell-1}\left(A_{k-\ell+1}, B\right) & \rightarrow \operatorname{nExt}_{\mathcal{A}}^{\ell}\left(A_{k-\ell}, B\right) \rightarrow \\
& \operatorname{nExt}_{\mathcal{A}}^{\ell}\left(M_{(k-\ell) n+1}, B\right)=0 .
\end{aligned}
$$

The claim follows.

Second, let us show that $\mathrm{nExt}_{\mathcal{A}}^{1}\left(A_{k-1}, B\right)$ is isomorphic to the cohomology of the complex (3) at $\mathcal{A}\left(M_{k n+1}, B\right)$. The conclusion follows from the commutative diagram

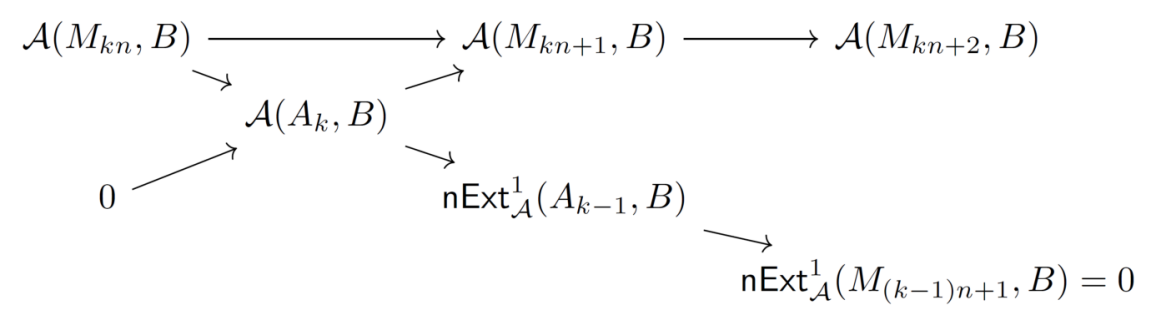

Theorem 3.4. Let $\mathcal{A}$ be an injectively cogenerated n-abelian category and $\mathcal{M}$ be an $(m, n)$-cluster tilting subcategory of $\mathcal{A}$. Then, $\mathcal{M}$ is an mn-abelian category.

Proof. We shall show that $\mathcal{M}$ satisfies the axioms of $m n$-abelian category.

(A0) Since the $n$-abelian category $\mathcal{A}$ is idempotent complete, it follows immediately from the definition of $(m, n)$-cluster tilting subcategory that $\mathcal{M}$ also is idempotent complete. 
(A1) Let $d^{0}: X^{0} \rightarrow X^{1}$ be a morphism in $\mathcal{M}$. Let $X^{1} \rightarrow \cdots \rightarrow X^{n+1}$ be an $n$-cokernel of $d^{0}$, applying Lemma 3.2 to $X^{n+1}$ gives the desired $m n$-cokernel of $d^{0}$. By duality, $d^{0}$ has an $m n$-kernel.

(A2) and $\left(\mathrm{A} 2^{o p}\right)$ Let $f^{0}: X^{0} \rightarrow X^{1}$ be a monomorphism in $\mathcal{A}$ such that $X^{0}, X^{1} \in \mathcal{M}$ and let $\left(f^{k}: X^{k} \rightarrow X^{k+1} \mid 1 \leq k \leq m n\right)$ be an $m n$-cokernel of $f^{0}$ in $\mathcal{M}$ obtained as in the previous paragraph. Applying the dual of Lemma 3.3 to $\left(f^{k}: X^{k} \rightarrow X^{k+1} \mid 0 \leq k \leq m n\right)$, we obtain that for all $Y \in \mathcal{M}$ and for all $k \in\{1, \ldots, m-1\}$ the cohomology of the induced complex

$$
\mathcal{A}\left(Y, X^{1}\right) \rightarrow \cdots \rightarrow \mathcal{A}\left(Y, X^{m n}\right) \rightarrow \mathcal{A}\left(Y, X^{m n+1}\right)
$$

at $\mathcal{A}\left(Y, X^{(k+1) n+1}\right)$ is isomorphic to $\mathrm{nExt}_{\mathcal{A}}^{k}\left(Y, X^{0}\right)$ which vanishes since $\mathcal{M}$ is an $(m, n)$-cluster-tilting subcategory of $\mathcal{A}$, cohomology of the sequence vanisher at $\mathcal{A}\left(Y, X^{j}\right)$ for $j \neq(k+1) n+1$. This shows that $\left(f^{0}, \ldots, f^{m n-1}\right)$ is an $m n$-kernel of $f^{m n}$ in $\mathcal{M}$. $\mathcal{M}$ also satisfies axiom $\left(\mathrm{A} 2^{o p}\right)$ follows by duality.

Proposition 3.5. Let $\mathcal{A}$ be an injectively cogenerated n-abelian category and $\mathcal{M}$ be an $(m, n)$-cluster tilting subcategory of $\mathcal{A}$. Then the sequence of morphisms

$$
X: X^{0} \rightarrow X^{1} \rightarrow \cdots \rightarrow X^{m n+1}
$$

is an mn-exact sequence of $\mathcal{M}$ if and only if is an $m$-fold $n$-exact sequence of $\mathcal{A}$. 
Proof. $(\Rightarrow)$. We split $d^{i n}: X^{i n} \rightarrow X^{i n+1}$ to $X^{i n} \stackrel{\pi^{i}}{\rightarrow} C^{i} \stackrel{j^{i}}{\rightarrow} X^{i n+1}$ such that $\pi^{i}$ is a cokernel of $d^{i n-1}$ for $i \in\{1,2, \cdots, m-1\}$. Then we show that $C^{i-1} \stackrel{j^{i-1}}{\rightarrow} X^{(i-1) n+1} \rightarrow \cdots \rightarrow X^{i n} \stackrel{\pi^{i}}{\rightarrow} C^{i}$ are $n$-exact sequence of $\mathcal{A}$, it is enough to prove $j^{i-1}$ are monomorphisms and $\mathrm{nExt}_{\mathcal{A}}^{j}\left(M, C^{i}\right)=0$ for any $i \in\{1,2, \cdots, m\}, j \in\{1,2, \cdots, m-1-i\}$ by induction, where $C^{0}=X^{0}$, $C^{m}=X^{m n+1}, j^{0}=d^{0}$ and $\pi^{m}=d^{m n}$.

For $i=1, j^{0}$ is a monomorphism. Applying $\mathcal{A}(M,-)$ on $X^{0} \rightarrow X^{1}$ $\rightarrow \cdots \rightarrow X^{n} \stackrel{\pi^{1}}{\rightarrow} C^{1}$, by long $n$-exact sequence theorem, we have $\mathrm{nExt}_{\mathcal{A}}^{j}\left(M, C^{1}\right)=0$ for $j \in\{1,2, \cdots, m-2\}$ since $\mathrm{nExt}_{\mathcal{A}}^{j}\left(M, X^{s}\right)=0$ for $s \in\{0,1, \cdots, n\}, j \in\{1,2, \cdots, m-1\}$. Suppose that $1 \leq k \leq m-1$ and that for all $\ell \leq k-1, j^{\ell}$ are monomorphisms and $\mathrm{nExt}_{\mathcal{A}}^{j}\left(M, C^{\ell+1}\right)=0$ for $j \in\{1,2, \cdots, m-2-\ell\}$. For $j^{k}$, let $u: A \rightarrow C^{k}$ be a morphism in $\mathcal{A}$ such that $j^{k} u=0$. Taking a right $\mathcal{M}$-approximation $v: M \rightarrow A \quad(v$ is epic since $\mathcal{M}$ is generating). Applying $\mathcal{A}(M,-)$ to $n$-exact sequence $C^{k-1} \rightarrow X^{(k-1) n+1} \rightarrow \cdots \rightarrow X^{k n} \rightarrow C^{k}$, by long $n$-exact sequence theorem, we have an exact sequence of groups

$$
\begin{aligned}
0 \rightarrow \mathcal{A}\left(M, C^{k-1}\right) \rightarrow \mathcal{A}\left(M, X^{(k-1) n+1}\right) & \rightarrow \cdots \rightarrow \mathcal{A}\left(M, X^{k n}\right) \rightarrow \mathcal{A}\left(M, C^{k}\right) \\
& \rightarrow \operatorname{nExt}_{\mathcal{A}}^{1}\left(M, C^{k-1}\right)=0
\end{aligned}
$$

Then there exists a morphism $w: M \rightarrow X^{k n}$ such that $u \nu=\pi^{k} w$, we have

$$
d^{k n} w=j^{k} \pi^{k} w=j^{k} u \nu=0
$$


Therefore, since $d^{k n-1}$ is a weak kernel of $d^{k n}$, there exists a morphism $s: M \rightarrow X^{k n-1}$ such that $d^{k n-1} s=w$, thus $u \nu=\pi^{k} d^{k n-1} s=0, u=0$, since $v$ is an epimorphism, this provides that $j^{k}$ is a monomorphism. It follows that

$$
C^{k} \rightarrow X^{k n+1} \rightarrow \cdots \rightarrow X^{(k+1) n} \rightarrow C^{k+1}
$$

is an $n$-exact sequence. Applying $\mathcal{A}(M,-)$ to (5), by long $n$-exact sequence theorem, we have exact sequence of groups for $j=1,2, \cdots, m-2$

$$
\begin{array}{r}
0=\mathrm{nExt}_{\mathcal{A}}^{j}\left(M, X^{(k+1) n}\right) \rightarrow \mathrm{nExt}_{\mathcal{A}}^{j}\left(M, C^{k+1}\right) \rightarrow \mathrm{nExt}_{\mathcal{A}}^{j+1}\left(M, C^{k}\right) \rightarrow \mathrm{nExt}_{\mathcal{A}}^{j+1} \\
\left(M, X^{k n+1}\right)=0 .
\end{array}
$$

but, $\quad \mathrm{nExt}_{\mathcal{A}}^{j+1}\left(M, C^{k}\right)=0$ for $j=0, \cdots, m-2-k$, this finishes the induction steps.

$(\Leftarrow)$. We split $X$ to $m n$-exact sequences

$$
C^{i-1} \stackrel{j^{i-1}}{\rightarrow} X^{(i-1) n+1} \rightarrow \cdots \rightarrow X^{i n} \stackrel{\pi^{i}}{\rightarrow} C^{i}
$$

where $C^{0}=X^{0}, C^{m}=X^{m n+1}, j^{0}=d^{0}$ and $\pi^{m}=d^{m n}$ for $i=1,2, \cdots, m$. Applying $\mathcal{A}(M,-)$ to these $n$-exact sequences, by long $n$-exact sequence theorem, it is easily prove that $\mathrm{nExt}_{\mathcal{A}}^{1}\left(\mathcal{M}, C^{j}\right)=0$ for $j=0,1, \cdots, m-2$.

We only need to show that $d^{i n}$ is a weak kernel of $d^{i n+1}$ and $d^{i n}$ is a weak cokernel of $d^{i n-1}$ for $i=1,2, \cdots, m-1$. We only show that $d^{i n}$ is a weak kernel of $d^{i n+1}$. Let $u: M \rightarrow X^{i n+1}$ be a morphism in $\mathcal{M}$ such that $d^{i n+1} u=0$, since $j^{i}$ is a kernel of $d^{i n+1}$, there exists a morphism $\nu: M \rightarrow C^{i}$ such that $j^{i} v=u$. Applying $\mathcal{A}(M,-)$ to $n$-exact sequence $C^{i-1} \rightarrow X^{(i-1) n+1} \rightarrow \cdots \rightarrow X^{i n} \rightarrow C^{i}$, by long $n$-exact sequence theorem, we have an exact sequence of groups like (4), thus, there exists a morphism $w: M \rightarrow X^{i n}$ such that $\pi^{i} w=\nu$, so $d^{i n} w=u$. 
Theorem 3.6. Let $\mathcal{A}$ be a projectively generated abelian category, $\mathcal{D}$ be an n-cluster tilting subcategory of $\mathcal{A}$ which closed under n-th syzygy, and $\mathcal{M}$ be an additive full subcategory of $\mathcal{D}$. Then, if $\mathcal{M}$ is an mn-cluster tilting subcategory of $\mathcal{A}$, then $\mathcal{M}$ is an $(m, n)$-cluster tilting subcategory of $\mathcal{D}$.

Proof. If $\mathcal{M}$ is an $m n$-cluster tilting subcategory of $\mathcal{A}$, then

$$
\begin{aligned}
\mathcal{M} & =\left\{X \in \mathcal{A} \mid \forall i \in\{1,2, \ldots, m n-1\} \operatorname{Ext}_{\mathcal{A}}^{i}(X, \mathcal{M})=0\right\} \\
& =\left\{X \in \mathcal{D} \mid \forall i \in\{1,2, \ldots, m n-1\} \operatorname{Ext}_{\mathcal{A}}^{i}(X, \mathcal{M})=0\right\}
\end{aligned}
$$

but, $\mathcal{D}$ is an $n$-cluster tilting subcategory closed under $n$-th syzygy, so $\mathcal{D}$ is an $n$-abelian category which has enough projective objects, by Lemma 2.3, we have $\operatorname{Ext}_{\mathcal{A}}^{j}(X, \mathcal{M})=0$ for all $j \neq k n, k \in \mathbb{N}^{*}$, so

$$
\begin{aligned}
\{X \in \mathcal{D} \mid & \left.\forall i \in\{1,2, \ldots, m n-1\} \operatorname{Ext}_{\mathcal{A}}^{i}(X, \mathcal{M})=0\right\} \\
= & \left\{X \in \mathcal{D} \mid \forall i \in\{1,2, \ldots, m-1\} \operatorname{Ext}_{\mathcal{A}}^{i n}(X, \mathcal{M})=0\right\} \\
= & \left\{X \in \mathcal{D} \mid \forall i \in\{1, \ldots, m-1\} \operatorname{nExt}_{\mathcal{D}}^{i}(X, \mathcal{M})=0\right\} .
\end{aligned}
$$

Since $\mathcal{M}$ is generating and cogenerating functorial finite subcategory of $\mathcal{A}$, so is generating and cogenerating functorial finite subcategory of $\mathcal{D}$. So, $\mathcal{M}$ is an $(m, n)$-cluster tilting subcategory of $\mathcal{D}$.

\section{Acknowledgement}

This work was supported by the Natural Science Foundation of China (CN) \#11901191, by the Scientific Research Fund of Hunan Provincial Education Department (CN) \#18C0639, and by the Study Learning and innovative experiment project for college students in Hunan Province\#S201910543024. 


\section{References}

[1] Aslak Bakke Buan, Robert Marsh, Markus Reineke, Idun Reiten and Gordana Todorov, Tilting theory and cluster combinatorics, Advances in Mathematics 204(2) (2006), 572-618.

DOI: https://doi.org/10.1016/j.aim.2005.06.003

[2] Osamu Iyama, Higher-dimensional Auslander-Reiten theory on maximal orthogonal subcategories, Advances in Mathematics 210(1) (2007), 22-50.

DOI: https://doi.org/10.1016/j.aim.2006.06.002

[3] Osamu Iyama, Cluster tilting for higher Auslander algebras, Advances in Mathematics 226(1) (2011), 1-61.

DOI: https://doi.org/10.1016/j.aim.2010.03.004

[4] Osamu Iyama and Yuji Yoshino, Mutation in triangulated categories and rigid Cohen-Macaulay modules, Inventiones Mathematicae 172(1) (2008), 117-168.

DOI: https://doi.org/10.1007/s00222-007-0096-4

[5] Gustavo Jasso, $n$-abelian and $n$-exact categories, Mathematische Zeitschrift 283(3-4) (2016), 703-759.

DOI: https://doi.org/10.1007/s00209-016-1619-8

[6] S. Kvamme, Projectively generated $d$-abelian categories are $d$-cluster tilting, arXiv: $1608.07985 \mathrm{v} 3$.

[7] Christof Geiss, Bernhard Keller and Steffen Oppermann, $n$-angulated categories, Journal für die Reine und Angewandte Mathematik (Crelles Journal) 675 (2013), 101-120.

DOI: https://doi.org/10.1515/CRELLE.2011.177

[8] Deren Luo, Homological algebra in $n$-abelian categories, Proceedings-Mathematical Sciences 127(4) (2017), 625-656.

DOI: https://doi.org/10.1007/s12044-017-0345-4

[9] Deren Luo and Panyue Zhou, The long $n$-exact sequence theorem in $n$-abelian categories, arXiv:2003.01493. 